\title{
PRODUÇÃO E COMPOSIÇÃO DOS RESÍDUOS DE SERVIÇO DE SAÚDE EM HOSPITAIS DO VALE DO ITAJAÍ
}

\author{
Sherelee Ribeiro Spindola ${ }^{1}$ e João Marcos Bosi Mendonça de Moura ${ }^{2}$
}

\begin{abstract}
Resumo: Conhecer a quantidade e a composição dos resíduos de serviço de saúde (RSS) é fundamental para a implementação de planos e sistemas de gestão nos estabelecimentos geradores. Por isso, objetivouse analisar a produção e composição dos RSS de hospitais da região do vale do Itajaí, estado de Santa Catarina, Brasil. Treze hospitais foram analisados e a quantidade de RSS foi correlacionada com o número de leitos ocupados. A taxa média de produção de RSS foi de 6,5 kg.leito ocupado ${ }^{-1}$.dia ${ }^{-1}$, com $5,4 \mathrm{~kg}$.leito

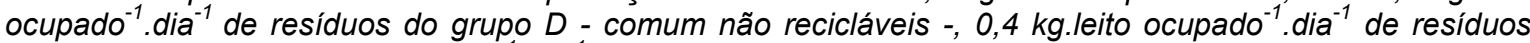
recicláveis e $0,7 \mathrm{~kg}$.leito ocupado ${ }^{-1} \cdot$ dia $^{-1}$ de resíduos perigosos (grupo $A, B$ e E). A correlação entre a produção de RSS e o número de camas ocupadas foi alta, com um coeficiente de correlação de 0,99 tanto para resíduos comuns quanto recicláveis, bem como para resíduos perigosos. A composição dos RSS é constituída por $49 \%$ de resíduos orgânicos, $23 \%$ de recicláveis e $28 \%$ de perigosos. Os resultados obtidos podem subsidiar os gestores no monitoramento e implantação de melhorias das políticas ambientais dos hospitais, além de poderem contribuir na redução dos custos operacionais relacionados aos resíduos.
\end{abstract}

Palavras-Chave: Resíduos sólidos. Reciclável. Infectantes. Geração. Composição gravimétrica.

\section{Introdução}

Os resíduos de serviço de saúde (RSS) representam uma preocupação para os hospitais devido ao seu risco à saúde pública e ao meio ambiente. Por isso, tem sido crescente no Brasil o desenvolvimento de sistemas integrados de gestão de RSS. Busca-se fortalecer as políticas de redução da produção de resíduos e os projetos de melhoria das condições de destinação final (PIAZZA; GOHR PINHEIRO, 2015).

Contudo, informações que abordem aspectos quali-quantitativos dos RSS, e subsidiem os gestores do sistema, ainda são pontuais no país e não conseguem ser extrapoladas para outras regiões. Vários estudos defendem que a primeira etapa para a implementação de planos e sistemas de gestão de RSS é o conhecimento da quantidade e da composição dos resíduos produzidos (ALTIN et al., 2003; KARAMOUZ et al., 2007; TAGHIPOUR; MOSAFERI, 2009). Maamari et al. (2015) afirmam que estes conhecimentos também auxiliam autoridades governamentais de fiscalização e licenciamento ambiental, que muitas vezes não dispõem de dados de referência.

Neste sentido, percebe-se a importância de estudos regionalizados que caracterizem a produção e a composição dos RSS (TESFAHUN; KUMIE; BEYENE, 2016).
Maders e Cunha (2015) determinaram uma taxa de geração de RSS igual a $5,2 \mathrm{~kg}$. leito ocupado $^{-1} \cdot$ dia $^{-1}$ no hospital de Macapá, no estado do Amapá. Kist et al. (2016) observaram uma taxa de geração de RSS do Grupo A, B e E igual a $0,47 \mathrm{~kg}$. leito $^{-1} \cdot \mathrm{dia}^{-1}$ em um hospital no interior do Rio Grande do Sul. André, Veiga e Takayanagui (2016) estudaram a geração de RSS em 11 hospitais do município de Ribeirão Preto, São Paulo. Observou-se a produção média de $7814,98 \mathrm{~kg} \mathrm{dia}^{-1}$ de RSS, sendo $67 \%$ resíduos comuns e $33 \%$ resíduos perigosos.

Em outros países há pesquisas que vão além, e correlacionam a quantidade de RSS com o número de leitos ocupados nas instituições (KOMILIS; KATSAFAROS; VASSILOPOULOS, 2011; MAAMARI et al., 2015 TESFAHUN; KUMIE; BEYENE, 2016). Moreira e Günther (2013) afirmam que é a maneira mais simplificada de predizer a quantidade de RSS gerado. Lemos, Silva e Pinto (2010) destacam que quando a produção de RSS é retratada em "kg.leito ocupado $^{-1}$ ", a produção de RSS dos hospitais possui chance de ser representada satisfatoriamente.

Sendo assim, o presente estudo objetivou analisar a produção e composição dos RSS de hospitais da região do vale do Itajaí, estado de Santa Catarina. Uma análise de correlação foi aplicada sobre os dados de

${ }^{1}$ E-mail: shere.lee@hotmail.com 


\section{REA - Revista de estudos ambientais (Online) v.18, n. 2, p.16-24, jul./dez. 2016}

produção de resíduos e de número de leitos ocupados. Buscou-se com o conhecimento gerado oferecer subsídios importantes para a gestão de RSS dos hospitais da região.

\section{Metodologia}

\section{1 Área de estudo}

A região do vale do Itajaí localiza-se na vertente atlântica do estado de Santa Catarina com aproximadamente 1,5 milhão de habitantes distribuídos em 59 municípios (IBGE, 2010). Dados da Vigilância Estadual registram 38 hospitais na região, sendo $47 \%$ com até 50 leitos, $37 \%$ com 51 a 150 leitos e $16 \%$ com 151 a 500 leitos disponíveis (SANTA CATARINA, 2012).

Atendimentos de alta complexidade são isolados, mas importantes, como os que ocorrem em Blumenau. O município localizase no médio vale do Itajaí e trata-se do mais populoso da região, com aproximadamente 343.715 habitantes (IBGE, 2016). Destacase nacionalmente por ser referência em transplante de coração, rim e fígado. $\mathrm{Na}$ metade de 2016 alcançou o milésimo transplante hepático. No início de 2015 registrou-se o marco histórico de 1000 transplantes renais (HSI, 2016).

\subsection{Coleta de dados}

Entre abril e agosto de 2016 foram procurados os 38 hospitais cadastrados pela Vigilância do Estado de Santa Catarina (SANTA CATARINA, 2012). Buscou-se o primeiro contato por telefone, com o(a) responsável técnico(a) pelo gerenciamento de RSS do hospital. Caso ocorressem três tentativas sem êxito, procurava-se a participação via e-mail.

Os dados foram fornecidos por correio eletrônico e respondiam as seguintes perguntas: a) qual a quantidade de resíduos gerada por ano no hospital?; b) qual o número de leitos disponíveis (LD)?; c) e qual o número médio de leitos ocupados (LO) no mesmo período de quantificação dos resíduos? Todas as respostas fornecidas corresponderam a informações do ano de 2015 e/ou 2016.

\subsection{Análise de dados}

Considerou-se a divisão dos RSS em cinco grupos, conforme a resolução $\mathrm{n}^{\circ} 306$ (ANVISA, 2004):

- Grupo A - resíduos infectantes, ou seja, com possível presença de agentes biológicos que podem apresentar risco de infecção;

- Grupo B - resíduos contendo substâncias químicas que podem apresentar risco à saúde pública e/ou ao meio ambiente, dependendo de suas características de inflamabilidade, corrosividade, reatividade e toxicidade;

- Grupo C - resíduos radioativos ou contaminados com radionuclídeos;

- Grupo D- resíduos que não apresentem risco biológico, químico ou radiológico à saúde e/ou ao meio ambiente, podendo ser equiparados aos resíduos domiciliares;

- Grupo E - materiais perfurocortantes ou escarificantes.

Consideraram-se resíduos perigosos aqueles pertencentes ao grupo $A, B$ e E.

Para a formulação das relações preditivas de produção de RSS, em função do $n^{\circ}$ de leitos ocupados, utilizou-se a regressão linear simples.

\section{Resultados e discussão}

\subsection{Caracterização dos hospitais}

Dos 38 hospitais, 22 responderam à pesquisa, sendo que 4 declararam não serem mais hospitais, 3 afirmaram que não quantificam os seus resíduos e 2 não quiseram participar. Ao todo, 13 hospitais informaram seus quantitativos, somando 1327 leitos com média de ocupação de $69 \%$ (921 leitos). A soma dos RSS registrados foi de 1795 toneladas anuais (Tabela 1).

Os 13 hospitais que quantificam seus RSS registram pelo menos a quantidade produzida de resíduos perigosos, ou seja, resíduos do grupo A, B e E (ANVISA, 2004). Nenhum dos hospitais participantes declarou a quantidade gerada de RSS do grupo C (radioativos). Observou-se que 6 quantificam também o RSS comum (grupo D), sendo que em 4 deles separa-se os resíduos recicláveis dos não recicláveis (Tabela 2 ). 


\section{REA - Revista de estudos ambientais (Online)} v.18, n. 2, p.16-24, jul./dez. 2016

Tabela 1 - Adesão à pesquisa e caracterização geral dos hospitais respondentes

\begin{tabular}{c|c|c|c|c}
\hline & $\begin{array}{c}\mathbf{N}^{\circ} \text { de } \\
\text { hospitais }\end{array}$ & $\begin{array}{c}\mathbf{N}^{\circ} \text { de leitos } \\
\text { disponíveis }\end{array}$ & $\begin{array}{c}\mathbf{N}^{\circ} \text { de leitos } \\
\text { ocupados }\end{array}$ & $\begin{array}{c}\text { Soma dos } \\
\text { RSS } \\
\text { registrados } \\
\text { ton.ano }^{-1} \text { ) }\end{array}$ \\
\hline $\begin{array}{c}\text { Total } \\
\text { Respondentes }\end{array}$ & 38 & & \\
Quantificam & 13 & 1327 & 921 & 1795 \\
Não são mais hospitais & 4 & & & \\
Não quantificam & 3 & & \\
Não quiseram participar & 2 &
\end{tabular}

Fonte: Autores (2016)

Tabela 2 - Caracterização dos hospitais pesquisados

\begin{tabular}{|c|c|c|c|c|c|c|}
\hline \multirow[b]{2}{*}{ Hospital } & \multirow[b]{2}{*}{$\begin{array}{l}\text { Leitos } \\
\text { disponíveis }\end{array}$} & \multirow[b]{2}{*}{$\begin{array}{c}\text { Leitos } \\
\text { ocupados }\end{array}$} & \multirow[b]{2}{*}{$\begin{array}{l}\text { Ano base } \\
\text { dos dados }\end{array}$} & \multicolumn{3}{|c|}{ O que quantifica? } \\
\hline & & & & Perigosos $^{1}$ & $\begin{array}{c}\text { Comum² } \\
\text { (sem } \\
\text { separação } \\
\text { do } \\
\text { reciclável) }\end{array}$ & $\begin{array}{l}\text { Comum: } \\
\text { (com } \\
\text { separação } \\
\text { reciclável e } \\
\text { não } \\
\text { reciclável) }\end{array}$ \\
\hline 1 & 267 & 211 & 2015 & $x$ & $x$ & \\
\hline 2 & 250 & 213 & Mar 2015/ Fev 2016 & $x$ & & $x$ \\
\hline 3 & 188 & 124 & 2015 & $x$ & & $x$ \\
\hline 4 & 165 & 140 & 2015 & $x$ & & $x$ \\
\hline 5 & 100 & 54 & Jan/Set 2016 & $x$ & $x$ & \\
\hline 6 & 84 & 48 & 2015 & $x$ & & \\
\hline 7 & 78 & 27 & Jun 2016 & $x$ & & \\
\hline 8 & 70 & 33 & Jan/Set 2016 & $x$ & & \\
\hline 9 & 58 & 22 & Jun 2015/ Maio 2016 & $x$ & & \\
\hline 10 & 22 & 18 & 2015 & $x$ & & $x$ \\
\hline 11 & 22 & 15 & Jan/Set 2016 & $x$ & & \\
\hline 12 & 16 & 10 & 2015 & $x$ & & \\
\hline 13 & 7 & 4 & Jan/Set 2016 & $x$ & & \\
\hline Total & 1327 & 921 & & 13 & 2 & 4 \\
\hline
\end{tabular}

${ }^{1}$ Grupo A, B e E (ANVISA, 2004).

2 Grupo D (ANVISA, 2004).

Fonte: Autores (2016)

Quatro (4) hospitais responderam com base nos quantitativos gerados entre janeiro e setembro de 2016 , seis (6) com base ao ano completo de 2015, dois (2) com dados anuais que abrangeram dois anos distintos e um (1) cujos dados correspondem a um quantitativo mensal (Tabela 2 ).

\subsection{Produção}

\subsubsection{Taxa de produção de RSS}

Os hospitais do vale do Itajaí ("n") acumularam uma taxa de 0,7 kg.leito ocupado $^{-1} \cdot \mathrm{dia}^{-1}(\mathrm{n}=13)$ de resíduos perigosos (grupo A, B e E). Para os resíduos recicláveis observou-se a produção média de $0,4 \mathrm{~kg}$.leito ocupado ${ }^{-1} \cdot \mathrm{dia}^{-1}(\mathrm{n}=3)$, excluindose da quantificação o hospital que apresenta um grande refeitório, e que por isso, produz uma alta quantidade de resíduos recicláveis, possuindo portanto, uma realidade distinta dos demais. No caso dos resíduos do grupo D - comum não recicláveis o número observado foi de $5,4 \mathrm{~kg}$.leito ocupado ${ }^{-1} \cdot \mathrm{dia}^{-1}$ $(n=3)$. Portanto a taxa de produção de RSS nos hospitais analisados $(n=13)$ foi de 6,5 kg.leito ocupado ${ }^{-1} \cdot \mathrm{dia}^{-1}$.

Schneider et al. (2001) indicam que a 


\section{REA - Revista de estudos ambientais (Online) v.18, n. 2, p.16-24, jul./dez. 2016}

produção de RSS pode variar de região para região, conforme 0 seu grau de desenvolvimento econômico. Países desenvolvidos geram uma maior quantidade de RSS por leito ocupado devido à maior participação das atividades de alta complexidade, o uso crescente de materiais descartáveis e um maior atendimento à população idosa (LEMOS; SILVA; PINTO, 2010; WHO, 2014). Nos Estados Unidos, por exemplo, esta taxa varia de 7 a $10 \mathrm{~kg}$.leito ocupado $^{-1} \cdot \mathrm{dia}^{-1}$ (WHO, 2014). No Brasil esse número é estimado em $3,8 \mathrm{~kg}$.leito ocupado ${ }^{1}$.dia ${ }^{-1}$ (TIVIROLLI et al., 2012).

Percebe-se que o valor encontrado para os hospitais do vale do Itajaí encontram-se no limiar de taxas características de países desenvolvidos, como os Estados Unidos. Tal constatação corrobora com a realidade diversa da região. Por um lado dispõe de instituições que realizam atividades de média a alta complexidade e que empregam maior número de materiais descartáveis. Por outro, ainda há pequenos hospitais que realizam atividades de baixa complexidade.

Em relação às taxas individualizadas por grupos de RSS, percebe-se que os valores são semelhantes aos observados em 11 hospitais de Ribeirão Preto, São Paulo (ANDRÉ; VEIGA; TAKAYANAGUI, 2016). Observou-se nos hospitais do vale do Itajaí uma produção de resíduos do grupo $D$ (recicláveis + não recicláveis) igual a 5,8 kg.leito ocupado ${ }^{-1} \cdot$ dia $^{-1}$, enquanto que em Ribeirão Preto - SP, os valores ficaram entre 5,3 e 6,5 kg.leito ocupado ${ }^{-1} \cdot \mathrm{dia}^{-1}$ (ANDRÉ; VEIGA; TAKAYANAGUI, 2016).

Em 12 hospitais da Grécia a taxa média de RSS perigosos atingiu $0,85 \mathrm{~kg}$.leito ocupado $^{-1} \cdot \mathrm{dia}^{-1}$ (SANIDA et al., 2010), sendo que 11 deles possuem mais de 100 leitos. Trata-se de hospitais maiores que os 13 investigados na região do vale do Itajaí, na qual apenas 5 hospitais possuem 100 ou mais leitos. Por isso, a menor taxa de produção de resíduos perigosos dos hospitais do vale do Itajaí em comparação com o caso grego, provavelmente está associada ao menor número de internações e centros cirúrgicos por unidade de leito.

\subsubsection{Estimativas de produção de RSS}

Para os resíduos do grupo D (recicláveis + não recicláveis) utilizaram-se inicialmente todos os dados possíveis, ou seja, os dados de seis hospitais. Contudo, dois deles indicaram valores muito baixos, com taxas iguais a 1,2 e 2,2 kg.leito ocupado $^{-1} \cdot \mathrm{dia}^{-1}$ enquanto que os demais hospitais pesquisados e a literatura científica (ANDRÉ; VEIGA; TAKAYANAGUI, 2016) apresentam taxas próximas de $6,0 \mathrm{~kg}$.leito ocupado ${ }^{-1} \cdot \mathrm{dia}^{-1}$.

É pouco provável que a causa esteja no fato de que estes resíduos estejam sendo segregados como perigosos, visto que a produção de RSS perigosos destes dois hospitais é da mesma ordem de grandeza que dos demais. Provavelmente, a causa destes baixos índices valores é a de uma menor atenção que possa ter sido dada a quantificação deles, visto que não raras vezes, os resíduos do grupo $D$ são encaminhados para a coleta regular de resíduos sólidos domiciliares.

Deste modo, para os outros quatro hospitais (Figura 1a, n=4), a estimativa da quantidade de RSS do grupo $D$ pode ser determinada pela equação $1 \mathrm{com}$ um coeficiente de correlação de 0,99.

$r=2000 \cdot L O-5877$

onde, $r\left(\mathrm{~kg} \cdot \mathrm{ano}^{-1}\right)$ é a quantidade estimada de RSS do grupo $D$ a ser produzido por ano e $L O$ a quantidade média de leitos ocupados.

No caso dos resíduos perigosos (grupo A, B e E) utilizaram-se inicialmente dados de treze hospitais. Todavia, dois deles indicaram valores maiores em relação aos demais. Trata-se de um hospital de ensino e outro de natureza jurídica particular que apresentaram média de 5,8 kg.leito ocupado${ }^{1}$.dia ${ }^{-1}$, enquanto que os 11 restantes apresentaram uma taxa média de 0,6 kg.leito ocupado $^{-1} \cdot \mathrm{dia}^{-1}$. A maior produção de RSS perigosos em hospitais de ensino já foi identificada em outros estudos (WHO, 2014; ANDRÉ; VEIGA; TAKAYANAGUI, 2016). Ela pode ser causada pela grande rotatividade de professores, alunos e funcionários, o que dificulta o controle no manejo dos RSS (SCHNEIDER; PAIZ; STEDILE, 2012). Neste caso, a taxa de produção de RSS perigoso do hospital de ensino chegou aos expressivos $10 \mathrm{~kg}$.leito ocupado ${ }^{-1}$. $\mathrm{dia}^{-1}$. Por isso, não se garante representatividade da equação 2 para hospitais onde há ensino ou uma alta rotatividade de funcionários. 


\section{REA - Revista de estudos ambientais (Online)} v.18, n. 2, p.16-24, jul./dez. 2016

Figura 1 - Relação entre quantidade de RSS e $n^{\circ}$ de leitos ocupados nos hospitais do vale do Itajaí: a) Resíduo comum (grupo D); b) Resíduos perigosos (grupo A, B e E); c) Resíduos recicláveis

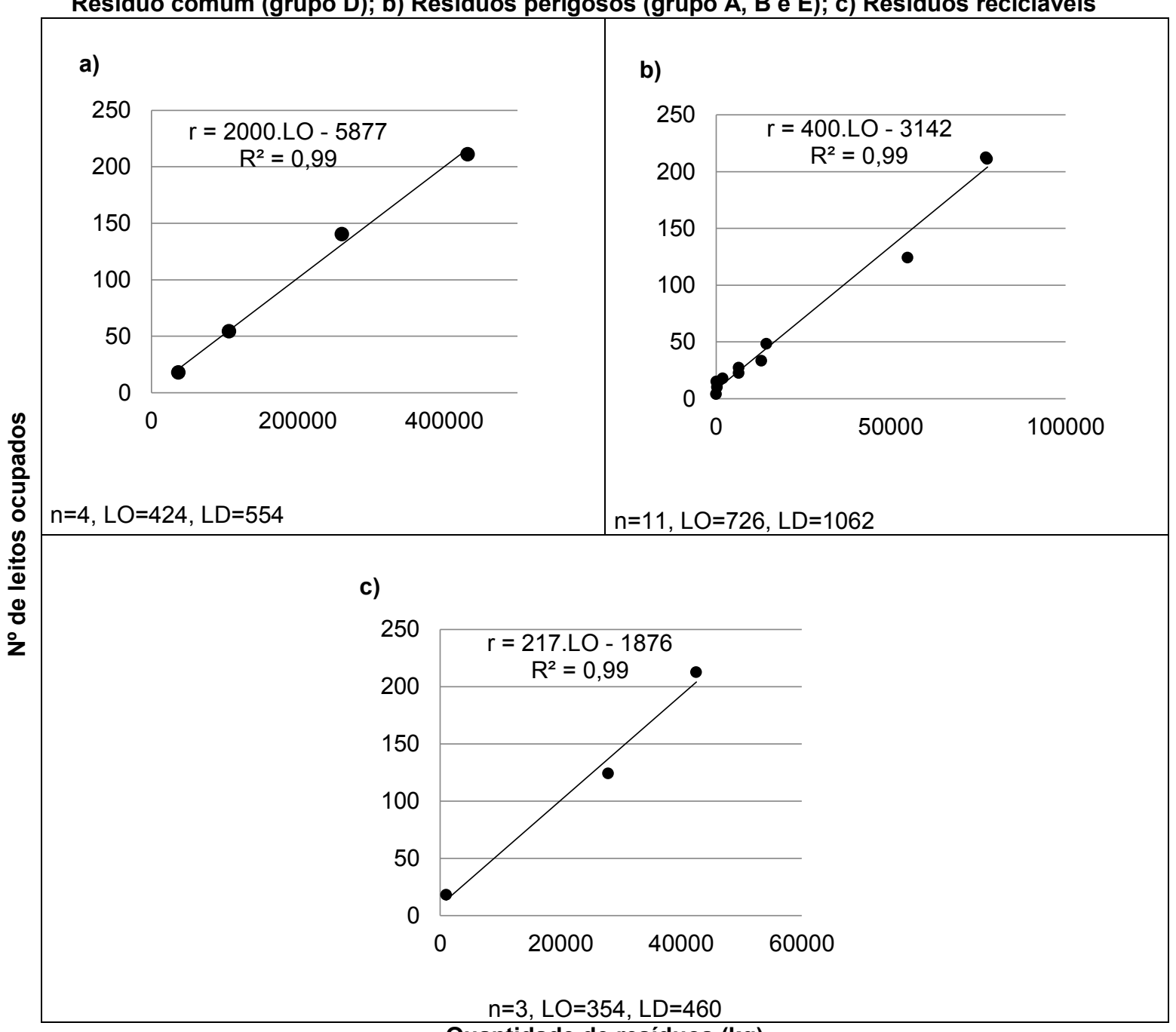

Quantidade de resíduos (kg)

$\mathrm{n}=$ quantidade de hospitais; $\mathrm{LO}=$ leitos ocupados; $\mathrm{LD}=$ leitos disponíveis

Fonte: Autores (2016)

No caso do hospital particular percebeu-se, que se aplicando as equações 1 e 2, a quantidade superestimada de RSS perigosos é semelhante a quantidade subestimada de resíduos comum (grupo D). Portanto, provavelmente importante parte dos resíduos comuns está sendo considerada perigosa. Observa-se quão útil pode ser essa abordagem global na identificação de possíveis fragilidades de gestão em estabelecimentos específicos. Essa realidade de falha na segregação é presente em alguns hospitais brasileiros (ADUAN et al. 2014; MOREIRA; YAMANE; SIMAN, 2015) o que representa custo maior de destinação final, ou seja, maior dispêndio de recursos da saúde.

Tendo em vista estes fenômenos pontuais no estudo, ambos os hospitais não foram inseridos na modelização (Figura 1b). Deste modo, observa-se a equação 2 que estima a quantidade de RSS perigosos (grupo A, B e E):

$$
r=400 \cdot L O-3142 \quad R^{2}=0,99
$$

onde, $r$ (kg.ano $^{-1}$ ) a quantidade estimada de RSS perigoso (grupo A, B e E) a ser produzido por ano e $L O$ a quantidade média de leitos ocupados.

Três hospitais pesquisados quantificam seus resíduos orgânicos, sendo que um possuía um refeitório grande comparado aos demais, com ocupação média de 450 colaboradores. Por esta razão, não se analisou a correlação porque seriam apenas dois hospitais.

Correlacionou-se, também, a 


\section{REA - Revista de estudos ambientais (Online) v.18, n. 2, p.16-24, jul./dez. 2016}

produção de RSS recicláveis com a quantidade de leitos ocupados (Figura 1c). Verificou-se que quatro hospitais quantificam seus resíduos recicláveis. Um hospital indicou uma alta produção. Trata-se do mesmo que se destacou pela grande produção de orgânicos, visto que possui uma contribuição importante do refeitório. Não obstante, os três restantes mantiveram uma tendência na produção de recicláveis em função do número de leitos ocupados (equação 3).

$$
r=217 \cdot L O-1876 \quad R^{2}=0,99
$$

Sendo, $r\left(\mathrm{~kg}^{\mathrm{ano}}{ }^{-1}\right)$ a quantidade estimada de recicláveis a ser produzida por ano e $L O$ a quantidade média de leitos ocupados.

\subsection{Composição}

Dados de cinco hospitais foram utilizados, sendo um excluído da análise por possuir uma composição com diferenças importantes comparada com os demais. Os outros sete restantes quantificam apenas os seus resíduos perigosos (Figura 2). Assim, a proporção observada foi de $72 \%$ de RSS Comum (grupo D) e $28 \%$ de RSS perigosos (grupo A, B e E).

Figura 2 - Composição dos RSS nos hospitais do vale do Itajaí ( $n=5, L O=706, L D=892)$

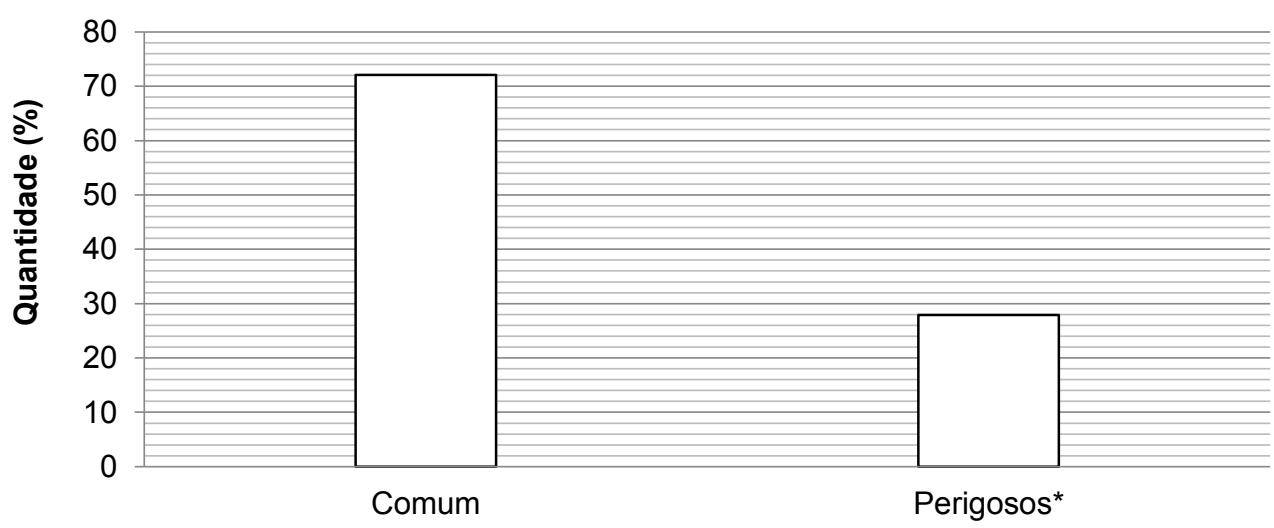

Tipo de resíduo

${ }^{*}$ GRUPO A, B e E (ANVISA, 2004).

$\mathrm{n}$ = quantidade de hospitais; $L O=$ leitos ocupados; $L D=$ leitos disponíveis

Fonte: Autores (2016)

Taghipour e Mosaferi (2009) ao estudarem 10 hospitais no Iran concluíram que $70 \%$ dos RSS eram comuns. André, Veiga e Takayanagui (2016) observaram que $67 \%$ dos RSS são comuns e $33 \%$ perigosos em hospitais paulistas de portes semelhantes a este estudo. Nota-se uma concordância dos resultados com a literatura científica.

Quando gerada uma composição gravimétrica mais específica (Figura 3), percebe-se que se mantém a proporção entre perigosos (grupo $A, B$ e $E$ ) e resíduos comuns (grupo D) e observa-se que dos resíduos comuns, $49 \%$ não são recicláveis e $23 \%$ os são.

Ao se observar que $49 \%$ de RSS não são recicláveis revela-se a importância de uma atenção especial ao gerenciamento dos resíduos não recicláveis, e em especial dos orgânicos em estabelecimentos hospitalares. Minimizar sua geração envolve a conscientização de funcionários e usuários/pacientes (TAGHIPOUR; MOSAFERI, 2009), integrando também os mais diversos estabelecimentos do hospital (clínicas, farmácia, refeitórios, restaurantes etc.).

Não menos importante é a quantidade de recicláveis $(23 \%)$, visto que podem representar dois impactos importantes sobre o sistema: primeiro negativo, caso não sejam (re)aproveitados, pois devido a sua baixa densidade, os recicláveis ocupam um volume maior por unidade de massa, e são os grandes responsáveis pela diminuição da vida útil de aterros sanitários. Por outro lado, impacto positivo é o fato de estes materiais serem (re)aproveitáveis com facilidade, inclusive, possibilitando a geração de renda para mitigar os custos do próprio sistema de gestão hospitalar (RIBEIRO; JUNIOR PISANI, 2012). 


\section{REA - Revista de estudos ambientais (Online) v.18, n. 2, p.16-24, jul./dez. 2016}

Figura 3 - Composição gravimétrica dos RSS em hospitais do vale do Itajaí (n=3, LO=477, LD=603)

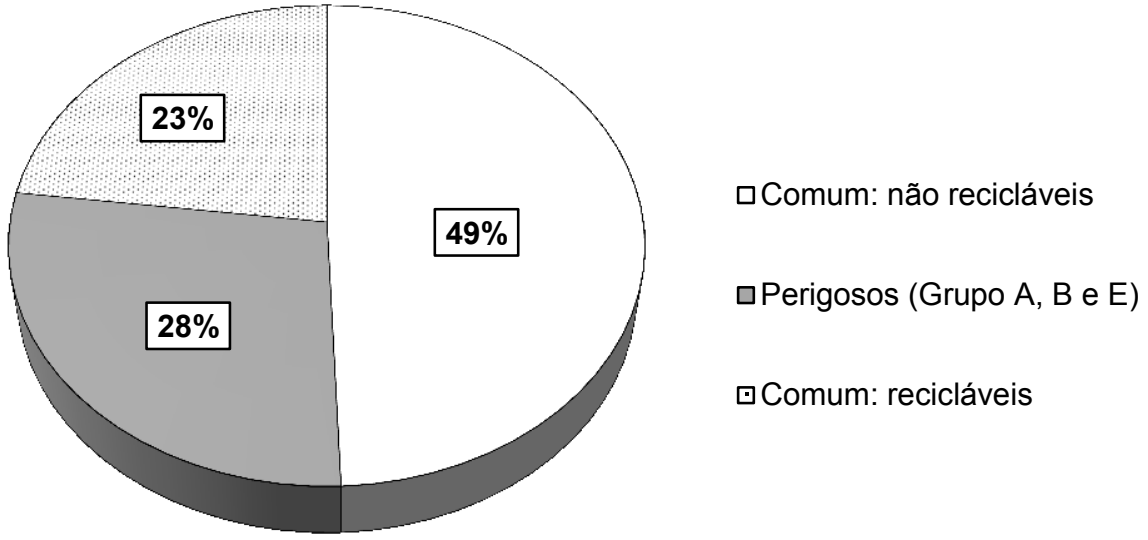

$\mathrm{n}$ = quantidade de hospitais; $\mathrm{LO}=$ leitos ocupados; $\mathrm{LD}=$ leitos disponíveis.

Fonte: Autores (2016)

Para Lemos, Silva e Pinto (2010) a maior produção dos RSS perigosos em hospitais está relacionada à existência de centros cirúrgicos. A Organização Mundial da Saúde estima que os RSS perigosos (hazardous health care waste) representem em média $15 \%$ do total gerado em hospitais (WHO, 2014). Observa-se que o valor obtido para os hospitais da região é maior. Uma das possíveis causas para essa diferença pode ser atribuída ao fato de que parte dos resíduos comuns são segregados como se fossem perigosos. É possível reduzir essa produção por meio de uma educação continuada, incorporada à capacitação profissional, possibilitando a redução de custos com tratamento/disposição final e a diminuição dos impactos ambientais negativos (MOREIRA; YAMANE; SIMAN, 2015).

\section{Conclusões e perspectivas}

Os hospitais da região do vale do Itajaí possuem taxas de produção e composição gravimétrica semelhante à descrita na literatura científica. A análise gravimétrica apontou que, praticamente, a metade dos RSS são (re)aproveitáveis. A análise global da produção de RSS possibilitou demonstrar, por meio de dados quantitativos, que estabelecimentos estejam provavelmente segregando parte dos seus resíduos comuns juntamente com os resíduos perigosos.

No desenvolvimento de modelos preditivos de produção de RSS deve-se salientar que estes são aplicáveis a hospitais com determinadas características estruturais. No caso da produção de comuns e recicláveis espera-se que o modelo explique melhor a realidade de estabelecimentos sem grandes contribuições de refeitórios, pois caso contrário, a relação "kg por leito ocupado" será pouco explicativa. $\mathrm{Na}$ quantificação de RSS perigosos, recomendam-se a formulação de outras análises para hospitais com caráter de ensino ou com alta rotatividade de funcionários (em fase de aprendizagem de segregação dos RSS).

Deste modo, espera-se que o estudo possibilite contribuir para o aperfeiçoamento dos sistemas de gestão de RSS voltados aos hospitais. Os dados obtidos nesta pesquisa podem subsidiar programas de monitoramento da produção de RSS, fortalecer programas de educação permanente, fundamentar avaliações de impactos ambientais, apoiar a redução de custos operacionais e, por fim, diminuir riscos à saúde pública e ao meio ambiente.

Para futuras pesquisas sugere-se a validação dos modelos de produção de RSS em outros hospitais. Novos trabalhos poderão avançar ao correlacionar a produção de RSS com o porte do hospital ou o número de pacientes em ambulatórios e em internações (outpatients/inpatients). Outros tipos de estabelecimentos de saúde poderiam também ser investigados e a produção/composição de RSS relacionada com a efetividade de seus Planos de Gerenciamento e o nível de conscientização ambiental dos seus colaboradores. 


\title{
REA - Revista de estudos ambientais (Online) v.18, n. 2, p.16-24, jul./dez. 2016
}

\section{Production and Composition of Health Care Waste in Hospitals of Itajaí Valley}

\begin{abstract}
The amount and composition of Health Care Waste (HCW) is a fundamental knowledge to the implementation of plans and management systems. Therefore, this study aimed to analyze the production and composition of HCW of hospitals in the region of Itajai valley, Santa Catarina state, Brazil. Thirteen (13) hospitals were analyzed and the amount of HCW was correlated with the number of occupied beds. The average rate of HCW production was $6.5 \mathrm{~kg}$. occupied bed ${ }^{-1}$. day ${ }^{-1}$, with $5.4 \mathrm{~kg}$. occupied bed ${ }^{-1}$. day ${ }^{-1}$ of nonrecyclable waste, $0.4 \mathrm{~kg}$. occupied bed ${ }^{-1}$. day ${ }^{-1}$ of recyclable waste and $0.7 \mathrm{~kg}$. occupied bed ${ }^{-1}$.day ${ }^{-1}$ of hazardous HCW. The correlation between the production of RSS and the number of occupied beds was high, with a correlation coefficient of 0.99 for both common and recyclable as well as for hazardous waste. The composition of the HCW is constituted by $49 \%$ organics waste, $23 \%$ recyclables and $28 \%$ hazardous waste. The obtained results can support the managers in the monitoring and implantation of improvements of the environmental policies of the hospitals, besides they can contribute in the reduction of the operational costs related to the waste.
\end{abstract}

Keywords: Solid waste. Recyclable. Infectious. Generation. Gravimetric composition.

\section{Referências}

ADUAN, S. A.; BRAGA, F. S.; ZANDONADE, E.; SALLES, D.; CUSSIOL, N. A. M.; LANGE, L. C. Avaliação dos resíduos de serviços de saúde do Grupo A em hospitais de Vitória (ES), Brasil. Eng. sanit. ambient, v. 19, n. 2, p. 133-141, 2014.

ALTIN, S.; ALTIN, A.; ELEVLI, B.; CERIT, O. Determination of hospital waste composition and disposal methods: a case study. Polish Journal of Environmental Studies, v. 12, n. 2, p. 251255, 2003.

ANDRÉ, S. C. da S.; VEIGA, T. B.; TAKAYANAGUI, A. M. M. Geração de Resíduos de Serviços de Saúde em hospitais do município de Ribeirão Preto (SP), Brasil. Eng. sanit. ambient, v. 21, n. 1, p. 123-130, 2016.

ANVISA. Resolução n³06 de 7 de dezembro de 2004. Dispõe sobre o Regulamento Técnico para o gerenciamento de resíduos de serviços de saúde. Disponível em: < http://bvsms.saude.gov.br/bvs/saudelegis/anvisa/2 004/res0306_07_12_2004.html>. Acesso em: 21 mar. 2016.

HSI - HOSPITAL SANTA ISABEL. Transplantes (alta complexidade). 2016. Disponível em: <http://www.santaisabel.com.br/sobre/1005/transp lantes--alta-complexidade->. Acesso em: 02 set. 2016.

IBGE - Instituto Brasileiro de Geografia e Estatística. Censo demográfico - Santa Catarina. 2010. Disponível em: <http://cidades.ibge.gov.br/xtras/uf.php?lang=\&co duf $=42 \&$ search=santa-catarina $>$. Acesso em: 01 set. 2016.
Blumenau. 2016. Disponível em: <http://cidades.ibge.gov.br/xtras/perfil.php?codmu $\mathrm{n}=420240>$. Acesso em: 01 set. 2016.

KARAMOUZ, M.; ZAHRAIE, B.; KERACHIAN, R.; JAAFARZADEH, N.; MAHJOURI, N.Developing a master plan for hospital solid waste management: A case study. Waste Management, v. 27, n. 5, p. 626-638, 2007

KIST, L. T.; ROSA, F. R.; MACHADO, E. L.; MORAES, J. A. R.. Gerenciamento e quantificação dos resíduos dos serviços de saúde: Estudo de caso. Tecno-Lógica, v. 20, n. 2, p. 111-117, 2016

KOMILIS, D.; KATSAFAROS, N.; VASSILOPOULOS, P. Hazardous medical waste generation in Greece: case studies from medical facilities in Attica and from a small insular hospital. Waste Management \& Research, v. 29, n. 8, p. 807-814, 2011.

LEMOS, K. I. L.; SILVA, M. G. C.; PINTO, F. J. M. Produção de resíduos em hospitais públicos e filantrópicos no município de Fortaleza (CE). Rev Baiana Saúde Pública, v. 34 , n. 2, p. 321-32, 2010.

MAAMARI, O.; BRANDAM, C.; LTEIF, R.; SALAMEH, D. Health Care Waste generation rates and patterns: The case of Lebanon. Waste Management, v. 43, p. 550-554, 2015.

MADERS, G. R.; CUNHA, H. F. A. Análise da gestão e gerenciamento dos resíduos de serviços de saúde (RSS) do Hospital de Emergência de Macapá, Amapá, Brasil. Eng. sanit. ambient, v. 20, n. 3, p. 379-388, 2015. 


\section{REA - Revista de estudos ambientais (Online) v.18, n. 2, p.16-24, jul./dez. 2016}

MOREIRA, A. M. M.; GÜNTHER, W. M. R. Assessment of medical waste management at a primary health-care center in São Paulo, Brazil. Waste Management, v. 33 , n. 1, p. 162-167, 2013.

MOREIRA, I. G.; YAMANE, L. H.; SIMAN, R. R. Análise quali-quantitativa dos resíduos gerados em enfermaria do Hospital Santa Casa de Misericórdia de Vitória, Espírito Santo. Revista de Ciências Ambientais, v. 9, n. 2, p. p. 23-35, 2015.

PIAZZA, G. A.; GOHR PINHEIRO, I. Logística reversa e sua aplicação na gestão de resíduos de medicamentos domiciliares. Revista de Estudos Ambientais, v. 16, n. 2, p. 48-56, 2015.

RIBEIRO, A. B.; PISANI JÚNIOR, R. Método de obter a geração de resíduos de serviço de saúde para monitorar a execução do Plano de Gerenciamento de Resíduos em um hospital. Revista AIDIS de Ingeniería y Ciencias Ambientales, v. 5, n. 2, p. 11-27.

SANIDA, G.; KARAGIANNIDIS, A.; MAVIDOU, F.; VARTZOPOULOS, D.; CHATZOPOULOS, S. Assessing generated quantities of infectious medical wastes: A case study for a health region administration in Central Macedonia, Greece. Waste Management, v. 30, n. 3, p. 532-538, 2010.

SANTA CATARINA. Mapa dos hospitais de Santa Catarina. 2012. Diretoria de Vigilância Sanitária de SC. Secretária de Saúde do Estado de Santa Catarina. Disponível em: < http://www.vigilanciasanitaria.sc.gov.br/index.php/ download/category/227-capacitacao-do-roteiro-depadroes-de-conformidade-em-unidade-hospitalare-legislacoes>. Acesso em: 21 mar. 2016.

SCHNEIDER, V.E.; EMMERICH, R. C.; DUARTE, V. C.; ORLANDIN, S. M. Manual de

\begin{abstract}
gerenciamento de resíduos sólidos de serviços de saúde. São Paulo: Baliero, 2001.
\end{abstract}

SCHNEIDER, V.E.; PAIZ, J.C.; STEDILE, N.L.R. Geração de resíduos em um hospital de ensino e pesquisa em saúde. In: Congresso internacional de tecnologias para o meio ambiente. 2012. Anais... Bento Gonçalves: 2012.

TAGHIPOUR, H.; MOSAFERI, M. Characterization of medical waste from hospitals in Tabriz, Iran. Science of the total environment, v. 407 , n. 5 , p. 1527-1535, 2009.

TESFAHUN, E.; KUMIE, A.; BEYENE, A. Developing models for the prediction of hospital healthcare waste generation rate. Waste Management \& Research, v. 34 , n. 1, p. $75-80$, 2016.

TIVIROLLI, K.; TIVIROLLI, S. C.; LUZ, P. C.; FUJINO, L. B. V.; SHINZATO, M. P.; SKOWRONSKI, J.; GOMES, A. O.; VASCONCELOS, L. H. A.; HESS, S. C. Gerenciamento dos resíduos em três hospitais públicos do Mato Grosso do Sul, Brasil. Revista Brasileira em Promoção da Saúde, v. 23, n. 3, p. 213-220, 2012.

WHO - World Health Organization. Safe management of wastes from health-care activities. World Health Organization, Geneva, Switzerland. 2014. Disponivel em: <http://www.searo.who.int/srilanka/documents/saf e_management_of_wastes_from_healthcare_activ ities.pdf>. Acesso em: 07 set. $20 \overline{16}$.

\section{Agradecimentos}

Os autores agradecem aos hospitais que gentilmente cederam seus dados para esta pesquisa e à Coordenação de Aperfeiçoamento de Pessoal de Nível Superior (CAPES) pela concessão da bolsa de doutorado ao segundo autor. 